\title{
Oxidative stress markers in patients of psoriasis with metabolic syndrome
}

\author{
Saurabh Sharma ${ }^{1, *}$, Roopam Bassi ${ }^{2}$, Manpreet Kaur ${ }^{3}$ \\ ${ }^{\mathbf{1}, 2}$ Professor ${ }^{3}$ Junior Resident, ${ }^{1,3}$ Dept. of Dermatology Venereology \& Leprosy, ${ }^{2}$ Dept. of Physiology, Sri Guru Ram Das Institute \\ of Medical Sciences \& Research Amritsar, Punjab, India
}

*Corresponding Author:

Email: drsaurabhsharma@yahoo.co.in

\begin{abstract}
Introduction: Psoriasis is a chronic inflammatory, $\mathrm{T}$ cell mediated immune disorder characterized by the formation of erythematous scaly papules and plaques.

Objective: To do a comparative evaluation of the markers of oxidative stress, namely glutathione peroxidase (GP), malondialdehyde (MDA) and superoxide dismutase (SOD) in patients of psoriasis with metabolic syndrome (MS), patients of psoriasis without metabolic syndrome and normal healthy subjects.

Materials and Methods: 150 subjects in the age group of 18-70 years were included in the study; Group A consisted of 50 psoriasis patients with metabolic syndrome, Group B consisted of 50 psoriasis patients without metabolic syndrome while Group $\mathrm{C}$ had 50 normal healthy individuals. Thorough history was taken and clinical examination was done. The punch biopsy was performed for confirmation of the diagnosis of psoriasis. Serum markers of oxidative stress were measured in each case and these values were then statistically compared among all the groups.

Results: On comparison of the groups, the mean values of Superoxide dismutase and Glutathione peroxidase were least in Group A $(248.3 \mathrm{U} / \mathrm{ml}, 6105 \mathrm{U} / \mathrm{ml})$ followed by Group B $(280 \mathrm{U} / \mathrm{ml}, 6868.3 \mathrm{U} / \mathrm{ml})$ and were maximum in Group C (395 U/ml, $14,058.3$ $\mathrm{U} / \mathrm{ml})$ whereas the mean value of Malondialdehyde was maximum in Group A $(1.0 \mathrm{nmol} / \mathrm{ml})$ followed by Group B $(0.7 \mathrm{nmol} / \mathrm{ml})$ and Group C $(0.13 \mathrm{nmol} / \mathrm{ml})$. On comparing these values among different groups, all the values were highly significant $(\mathrm{p}<0.001)$ in all the groups except Superoxide dismutase and Glutathione peroxidase among Groups A and B.

Conclusions: Free radical mediated oxidative stress play an important role in the pathogenesis of psoriasis especially when it is associated with metabolic syndrome. Therefore, anti-oxidants can have a preventive as well as a therapeutic role in such patients.
\end{abstract}

Keywords: Glutathione peroxidase (GP), Malondialdehyde (MDA), Metabolic syndrome (MS), Oxidative stress (OS), Psoriasis, superoxide dismutase (SOD).

\section{Introduction}

Psoriasis is characterized by exaggerated and disordered epidermal cell proliferation and keratinization. Tumour necrosis factor alpha, dendritic cells and T-cells all contribute substantially to its pathogenesis. ${ }^{1,2}$

With increase in our understanding of the disease and its pathogenesis, several studies have linked psoriasis with increase in oxidative stress in the local cutaneous milieu. The Reactive oxygen species (ROS) can be generated by either endogenous processes or from exogenous sources. They can react with normal cellular products resulting in their damage, hence disturbing the normal metabolic processes. ${ }^{3,4}$

To protect the skin against this over-load of oxidant species, it contains a well-organised system of enzymes which work in a synergistic manner. They prevent the production of oxidation products, such as Malondialdehyde (MDA) and further release of proinflammatory mediators. But when this oxidative stress overwhelms the antioxidant capacity of the skin it leads to the modification of the cellular redox apparatus, further leading unto an alteration of cellular homeostasis and commencement of several degenerative processes. ${ }^{5,6}$ Antioxidants mainly include Glutathione Peroxidase (GP), Superoxide dismutase (SOD) and catalase (CAT). ${ }^{7}$
Lately, several systemic diseases are being recognised in association with psoriasis which mainly include metabolic syndrome. ${ }^{8,9}$ Metabolic syndrome is a constellation of lipid and non-lipid cardiovascular risk factors of metabolic origin. These co-morbidities in conjunction with psoriasis can be associated with significant impairment in health related quality of life. Therefore, early recognition and treatment is of paramount importance. ${ }^{10}$

\section{Materials and Methods}

The present case control study is undertaken in the Department of Dermatology, Venereology \& Leprosy in collaboration with the Department of Biochemistry, Sri Guru Ram Das Institute of Medical Sciences \& Research, Vallah, Sri Amritsar. The selection criteria was of patients attending the OPD in the Department of Dermatology, Venereology \& Leprosy, having biopsy proven Chronic Plaque Psoriasis (involving more than $10 \%$ of B.S.A) in the age group of 18-70 years. 150 subjects were included in the study which were divided into three groups: Group A comprised of 50 subjects having psoriasis with Metabolic Syndrome (MS) as per the adult treatment panel III criteria. Group B comprised of 50 subjects having psoriasis without metabolic syndrome. Group C comprised of 50 subjects which were normal healthy individuals age and sex matched from the general 
population without any previous history of psoriasis or metabolic syndrome. Patients were divided into three categories according to the severity of psoriasis i.e. Group 1: 10-30\%, Group 2: $30-50 \%$ and Group 3: $>50 \%$. Proforma and diet questionnaire were duly filled, only the subjects with adequate diet intake were included. Information about the procedure was given to the patient and written consent was taken in all the cases. Clearance from the ethical committee was taken.

Metabolic syndrome was diagnosed by Adult Treatment Panel III which defines metabolic syndrome as the presence of at least 3 of the following conditions:

1. Abdominal obesity [waist circumference $>102 \mathrm{~cm}$ (40 inches) in men; >88 cm (35 inches) in women]

2. Elevated serum triglycerides $[>150 \mathrm{mg} / \mathrm{dL}(1.7$ mmol/l) or under treatment]

3. Low HDL cholesterol [men <40 mg/dL (1 mmol/l); women $<50 \mathrm{mg} / \mathrm{dl}(1.3 \mathrm{mmol} / \mathrm{l})$ or under treatment]

4. Elevated blood pressure $(>130 / 85 \mathrm{~mm} \mathrm{Hg}$ or under treatment)

5. Elevated fasting glucose $(>110 \mathrm{mg} / \mathrm{dl}$ or under treatment). ${ }^{9}$

Exclusion Criteria: Pregnant women, patients with other risk factors for oxidative stress such as AIDS, tuberculosis, smoking and alcohol consumption, patients with hepatic or renal disease, psoriatic arthritis, gout, rheumatoid arthritis and those on antioxidant supplements. In Group B and C, any subject showing a feature of metabolic syndrome was excluded.

Investigations: Skin biopsy was done in subjects of Groups A and B using $5 \mathrm{~mm}$ punch under local anesthesia and specimen was sent for histopathological examination. Fasting blood samples were collected from all the 150 subjects. $5 \mathrm{ml}$ venous blood was taken in a dry disposable syringe under all aseptic precautions by venepuncture in the antecubital vein in a sterile, dry acid washed vial for biochemical analysis. Serum Superoxide dismutase levels were measured by Marklund and Marklund method, 1974 modified by Nandi and Chatterjee, 1988. ${ }^{11,12}$ Serum Glutathione peroxidase estimation was done by continuous spectrophotometric rate determination and serum Malondialdehyde levels were measured by the method described by Satoh, 1988. ${ }^{13,14}$ After performing these tests, the data was analysed statistically by one way ANOVA with Post Hoc Tukey, comparing mean values of all the variables between the two groups. The values were considered significant if the $\mathrm{p}$ value was $<0.05$ and highly significant if the $\mathrm{p}$ value was $<0.001$. SPSS 17.0 software was used.

\section{Results}

The mean age of the subjects in Group A was 38.7 years and in Group B it was 35.8 years whereas in Group $C$ the mean age was 39.6 years. The differences in the mean age of the three groups was statistically insignificant $(\mathrm{p}>0.05)$. Male to female ratio in Group A was 1.5:1 whereas in Group B it was $1.2: 1$. Slight male preponderance was seen in both the groups but the difference was insignificant. All the patients included in our study were of Chronic Plaque Psoriasis. Trunk was the most common site involved, it was present in $90 \%$ (90) of the patients.

According to the severity of psoriasis, Malondialdehyde showed maximum levels in Group 3 followed by Group 2 and Group 1. The differences were statistically significant whereas levels of Superoxide dismutase and Glutathione peroxide were found to be insignificant.

Table 1, Fig. 1A, 1B and 1C show the comparison of the values of Superoxide dismutase, Glutathione peroxidase and Malondialdehyde in all the three groups. The mean value of Superoxide dismutase and Glutathione peroxidase was least in Group A followed by Group B and was maximum in Group C whereas the mean value of Malondialdehyde was maximum in Group A followed by Group B and Group C.

On comparing the parameters in Group A and B, though the values of Superoxide dismutase and Glutathione peroxide were decreased in Group A but the difference between the two groups was insignificant. On the other hand, the increase in the levels of pro-oxidant Malondialdehyde was found to be highly significant with $\mathrm{p}$ value $<0.001$.

On comparing the values of Superoxide dismutase and Glutathione peroxidase in Groups A and B with Group $C$, the mean value of both the markers were decreased in Groups A and B and the difference was highly significant ( $p$ value $<0.001$ ). On the other hand, the mean levels of Malondialdehyde were found to increased in Groups A and B as compared to Group C and the difference was highly significant with $p$ value $<0.001$.

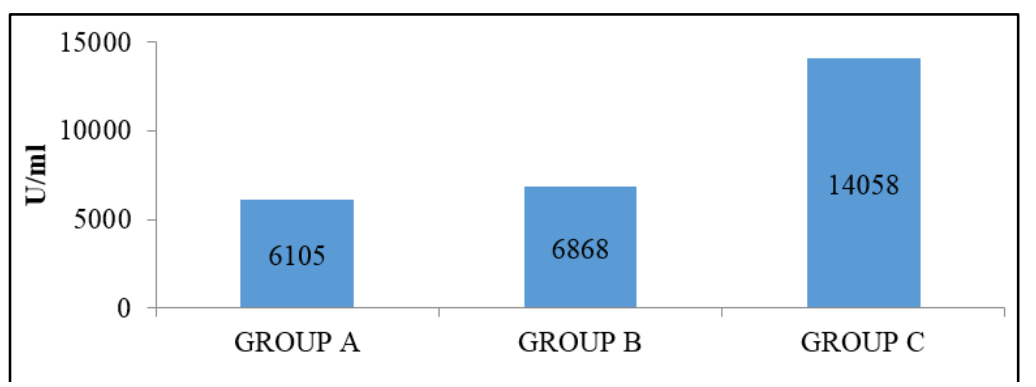

Fig. 1A: Comparison of glutathione peroxidase in all the three groups 


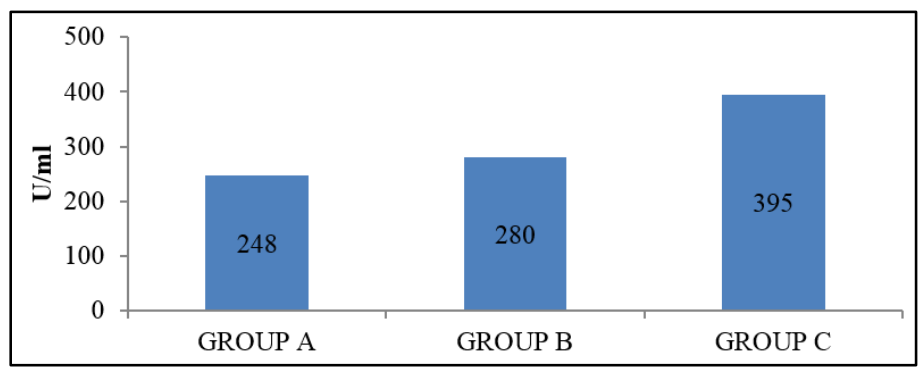

Fig. 1B: Comparison of superoxide dismutase in all the three groups

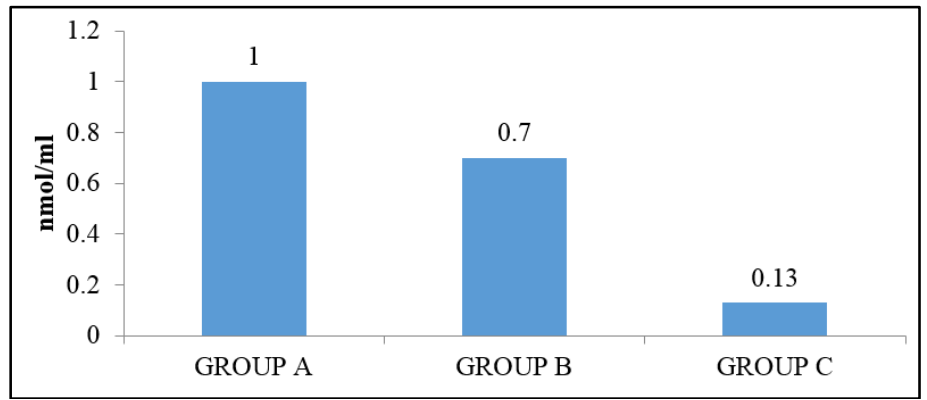

Fig. 1C: Comparison of malondialdehyde in all the three groups

Table 1: Comparison of mean levels of superoxide dismutase (SOD), glutathione peroxidase (GP) and malondialdehyde (MDA) in all the three groups

\begin{tabular}{|l|c|c|c|c|c|c|}
\hline \multicolumn{5}{|c|}{ Mean \pm SD } & \multicolumn{2}{c|}{ p value } \\
\hline Parameters & $\begin{array}{c}\text { Group A - } \\
\text { Mean } \pm \text { SD }\end{array}$ & $\begin{array}{c}\text { Group B - } \\
\text { Mean } \pm \text { SD }\end{array}$ & $\begin{array}{c}\text { Group C- } \\
\text { Mean } \pm \text { SD }\end{array}$ & A vs B & A vs C & B vs C \\
& $248.3 \pm$ & $280.46 \pm$ & $237.63 \pm$ & $>0.05^{*}$ & $<0.001^{* * *}$ & $<0.001^{* * *}$ \\
\hline SOD(U/ml) & 75.3 & 86.3 & 39.30 & & & \\
\hline GP $(\mathbf{U} / \mathbf{m l})$ & $6105 \pm$ & $6868.3 \pm$ & $1058 \pm$ & $>0.50^{*}$ & $<0.001^{* * *}$ & $<0.001^{* * *}$ \\
& 2509.3 & 3020.8 & 2329.1 & & & \\
\hline MDA(nmol/ml) & $1.0 \pm$ & $0.7 \pm$ & $0.13 \pm$ & $<0.001^{* * *}$ & $<0.001^{* * *}$ & $<0.001^{* * *}$ \\
& 1.0 & 0.21 & 0.1 & & & \\
\hline
\end{tabular}

$\mathrm{p}$ value $>0.05$ - not significant*, $\mathrm{p}$ value $<0.05$ - significant ${ }^{* *}, \mathrm{p}$ value $<0.001$ - highly significant $* * *$

\section{Discussion}

Oxidative stress represents a state of imbalance between the production and the consumption of the free radicals. In psoriasis, either there is increase in production of markers of oxidative stress due to the damaging forces or there are some obstacles in their consumption, leading to the initiation or aggravation of the disease process. Therefore, it becomes important to study these markers and to draw some valid conclusions. A few studies have been conducted on oxidative stress in psoriasis but on extensive research of literature we could not find any studies on the evaluation of oxidative stress in patients of psoriasis with metabolic syndrome. The present study was undertaken to resolve this existing lacuna in the prevailing literature.

In our study, the mean levels of Superoxide dismutase were decreased while Malondialdehyde levels were increased in Group A and B patients as compared to Group C. Similar findings have been observed by Priya R et al, in which on comparison of psoriasis patients with controls they found that the level of Malondialdehyde was significantly higher in cases when compared to controls $(p<0.0001)$ whereas the level of Superoxide dismutase and total anti-oxidant capacity (TAC) were significantly lower in cases as compared to controls $(\mathrm{p}<0.0001) .{ }^{15}$ In other study done by Pujari VM et al, they also observed raised Malondialdehyde levels in psoriasis patients as compared to controls with $\mathrm{p}$ value $<0.001 .^{16}$

In our study severity of the disease correlated with the levels of Malondialdehyde, similar findings have been observed by Sunitha $\mathrm{S}$ et al. ${ }^{17}$

Our study showed that the levels of another protective anti-oxidant Glutathione peroxidase were also decreased in patients of psoriasis alone and in patients of psoriasis with metabolic syndrome. Our results are in accordance with the study done by Wozniak et al in which they found that the mean levels of Glutathione peroxidase in psoriasis patients were decreased as compared to healthy individuals. ${ }^{18}$ 
Another study done by Bacchetti $\mathrm{T}$ et al showed that total antioxidant capacity is decreased in patients of Psoriasis. ${ }^{19}$

\section{Conclusion}

Our study provides an important evidence of considerable increase in the oxidative stress markers in both the patients of psoriasis with metabolic syndrome and in patients of psoriasis without metabolic syndrome. This may play a role in the pathogenesis of psoriasis. Therefore, attenuation of oxidative stress might be a relevant therapeutic approach in these patients and it can form an important part of futuristic and holistic management of psoriasis. It may also play some role in future diagnostics and monitoring of the response to treatment.

Additionally, all the patients of psoriasis with or without metabolic syndrome should be advised to inculcate healthy lifestyle changes with weight management strategies in their treatment regimen.

\section{References}

1. Griffiths CEM, Barker JNWN. Pathogenesis and clinical features of psoriasis. The Lancet. 2007;370(9583):26371.

2. MacDonald A, Burden AD. Psoriasis: advances in pathophysiology and management. Postgrad Med J. 2007;83(985):690-7.

3. Orient A, Donko A, Szabo A, Leto TL, Geiszt M. Novel sources of reactive oxygen species in the human body. Nephrol Dial Transplant. 2007;22:1281-8.

4. Bedard K, Krause KH. The NOX family of ROSgenerating NADPH oxidases: physiology and pathophysiology. Physiol Rev. 2007;87:245-313.

5. Briganti S, Picardo M. Antioxidant activity, lipid peroxidation and skin diseases. What's new. J Eur Acad Dermatol Venereol. 2003;17(6):663-9.

6. Finkelstein E, Rosen GM, Rauckmann GJ. Spin trapping of superoxide and hydroxyl radical: practical aspects. Arch Biochem Biophys. 1980;200: 1-16.

7. Vertuani S, Angusti A, Manfredini S. The antioxidants and pro-antioxidants network: an overview. Curr Pharm Des. 2004;10:1677-94.

8. Gottlieb AB, Chao C, Dann F. Psoriasis comorbidities. $J$ Dermatolog Treat. 2008;19(1):5-21.
9. Grundy SMCJ, Daniels SR, Donato KA, Eckel RH, Franklin BA, Gordon DJ, Krauss RM, Savage PJ, Smith SCJ, Spertus JA, Costa F. Diagnosis and management of the metabolic syndrome: an American Heart Association/National Heart, Lung and Blood Institute scientific statement. Curr Opin Cardiol. 2006;21(1):1-6.

10. Serrano EMY, Lista DJ, Orihuela PP, Martinez PP, Fuentes F, Marin C, Tunez I, Tinahones FJ, Jimenez FP, Roche HM, Miranda JL. Oxidative stress is associated with the number of components of metabolic syndrome: LIPGENE study. Experimental \& Molecular Medicine. 2013;45(1):28-30.

11. Marklund S, Marklund G. Involvement of superoxide anion radical in autooxidation of pyrogallol and a convenient assay of superoxide dismutases. Eur $J$ Biochem. 1974;47(3):469-74.

12. Nandi A, Chatterjee IB. Assay of superoxide dismutase activity in animal tissue. J. Biosci. 1988;13(3):305-15.

13. Wendel A. Enzymatic basis of detoxication. Academic Press. 1980;1:333.

14. Satoh K. Serum lipid peroxide in cerebrovascular disorders determined by a new colorimetric method. Clinica Chem Acta. 1978;90(1):37-43.

15. Priya R, Kumar U, Saran A, Kumari R, Kishore C. Oxidative stress in psoriasis. Biomedical Research. 2013;25(1):132-4.

16. Pujari VM, Ireddy S, Itagi I, Kumar S. The serum levels of malondialdehyde, vitamin e and erythrocyte catalase activity in psoriasis patients. J Clin Diagn Res. 2014;8(11):14-6.

17. Sunitha S, Rajappa M, Thappa DM, Chandrashekar L, Munisamy M, Revathy G, Priyadarssini M.

Comprehensive lipid tetrad index, atherogenic index and lipid peroxidation: Surrogate markers for increased cardiovascular risk in psoriasis. Indian J Dermatol Venereol Leprol. 2015;81:464-71.

18. Wozniak A, Drewa G, Krzyzynska ME, Czajkowski R, Protas DF, Mila KC, Rozwodowska M, Soponska M, Czarnecka ZE. Oxidant-antioxidant balance in patients with psoriasis. Med Sci Monit. 2007;13(1):30-3.

19. Bacchetti T, Campanati A, Ferretti G, Simonetti O, Liberati G, Offidani AM. Oxidative stress and psoriasis: the effect of antitumour necrosis factor- $\alpha$ inhibitor treatment. Br J Dermatol. 2013;168(5):984-9.

How to cite this article: Sharma S, Bassi R, Kaur M. Oxidative stress markers in patients of psoriasis with metabolic syndrome. Ind $\mathbf{J}$ Clin Exp Dermatol.2018;4(3):170-173. 